OPEN ACCESS

Edited by:

Alessia Ciarrocchi,

Local Health Authority of Reggio

Emilia, Italy

Reviewed by:

Simonetta Piana,

Sazienda USL-IRCCS

Reggio Emilia, Italy

Janete Maria Cerutti,

Federal University of São Paulo, Brazl

*Correspondence:

Qingzhi Liu

livqzh@scut.edu.cn

Bo Xu

eyboxu@scut.edu.cn

${ }^{\dagger}$ These authors share first authorship

Specialty section:

This article was submitted to

Thyroid Endocrinology,

a section of the journal

Frontiers in Endocrinology

Received: 01 March 2021 Accepted: 07 May 2021

Published: 23 June 2021

Citation:

Guo M, Chen Z, Li Y, Li S, Shen F, Gan X, Feng J, Cai W, Liu Q and Xu B (2021) Tumor Mutation Burden Predicts Relapse in Papillary Thyroid Carcinoma With Changes in Genes and Immune Microenvironment.

Front. Endocrinol. 12:674616. doi: 10.3389/fendo.2021.674616

\section{Tumor Mutation Burden Predicts Relapse in Papillary Thyroid Carcinoma With Changes in Genes and Immune Microenvironment}

\author{
Mengli Guo ${ }^{1 \dagger}$, Zhen Chen ${ }^{2 \dagger}$, Yayi $\mathrm{Li}^{2}$, Sijin $\mathrm{Li}^{2}$, Fei Shen ${ }^{1,2}$, Xiaoxiong Gan ${ }^{1,2}$, \\ Jianhua Feng ${ }^{1,2}$, Wensong Cai ${ }^{1,2}$, Qingzhi $\mathrm{Liu}^{3 *}$ and Bo Xu ${ }^{1,2 *}$

\footnotetext{
${ }^{1}$ Department of Thyroid Surgery, Guangzhou First People's Hospital, Guangzhou Medical University, Guangzhou, China, ${ }^{2}$ School of Medicine, South China University of Technology, Guangzhou, China, ${ }^{3}$ Chronic Disease Laboratory, Institutes for Life Sciences and School of Medicine, South China University of Technology, Guangzhou, China
}

Background: The risk factors of papillary thyroid carcinoma (PTC) recurrence are meaningful for patients and clinicians. Tumor mutation burden (TMB) has been a biomarker for the effectiveness of immune checkpoint inhibitor $(\mathrm{ICl})$ and prognosis in cancer. However, the role of TMB and its latent significance with immune cell infiltration in PTC are still unclear. Herein, we aimed to explore the effect of TMB on PTC prognosis.

Material and Methods: RNA-seq and DNA-seq datasets of PTC patients were downloaded from The Cancer Genome Atlas (TCGA) database. The Gene Ontology (GO) and gene set enrichment analysis (GSEA 4.0.1) were applied further to explore potential differences in PTC patients' biological functions. The differentially expressed genes (DEGs) and immune microenvironment between the high and low TMB groups were determined.

Results: TMB had the highest AUC score than other clinical indicators in ROC analysis on recurrence-free survival, and a higher TMB score was related to a worse prognosis. Further, GSEA showed a higher level of oxidative phosphorylation (OXPHOS) in the high TMB group, and four genes correlated with recurrence-free survival rate were identified. The abundance of $\mathrm{CD}^{+} \mathrm{T}$ cells and $\mathrm{M} 1$ macrophages in the high TMB group was significantly lower than that in the low TMB group.

Conclusions: Our study found that TMB was a better predictor variable at evaluating the risk of PTC recurrence. Moreover, TMB-related genes conferred dramatically correlated prognosis, which was worth exploring in guiding postoperative follow-up and predicting recurrence for PTC patients.

Keywords: papillary thyroid carcinoma (PTC), prognosis, The Cancer Genome Atlas (TCGA), tumor mutation burden (TMB), immune infiltrate 


\section{INTRODUCTION}

Papillary thyroid carcinoma (PTC) is the most common pathological type of thyroid carcinoma, with a good prognosis (1). Over the last four decades the occurrence of thyroid cancer was growing for the increased incidence of PTC, and the increase in mortality of thyroid cancer was mainly due to the increase in mortality of advanced-stage PTC (2).

Although the recurrence rate of some low-risk and intermediate-risk PTC patients undergoing hemithyroidectomy is low, the current risk scoring system has no predictive effect on these patients, which needs to be followed up to detect and treat the recurrence early (3). The aggressive clinical pathological, age $\geq 55$, male, tumor size $>4 \mathrm{~cm}$, tall cell variant, and positive lymph node metastasis are independent prognostic factors, and there may continue to be the risk of cancer death and early recurrence (4-7). Besides, many biomarkers for predicting the recurrence of PTC are reported, such as SMOC2 (8), Cyclin D1 and C-myc (9), serum fibrinogen levels (10), and $\mathrm{BRAF}^{\mathrm{V} 600 \mathrm{E}}$ mutation (11). However, most biomarkers have limitations or insufficient clinical applicability (12). It is imperative to find reliable prognostic markers for PTC.

Tumor mutation burden (TMB), which is a quantitative measurement of the total number of somatic non-synonymous mutations in each coding area of the tumor genome (13), was first reported to predict the activity of immune checkpoint inhibitor therapies in multiple cancers (14). The detection of TMB is based on massive next-generation sequencing (NGS) or whole-exome sequencing (15). In specific cancer types and ethnic groups, the number of driver mutations is significantly positively correlated with cancer incidence rates (16). One lung cancer research identified that specific mutations could result in tumorigenesis or recurrence, and patients with a high burden of tumor-specific gene mutations had poor recurrence-free survival (RFS) (17). Another study showed that further validation and research on the elevated TMB and the potential impact of tumorinitiating mutations in clinically unfavorable prostate cancer could improve the outcomes of patients (18). Higher TMB means more tumor neoantigens are exposed, so TMB has also become a predictor of response rate to immune checkpoint inhibitor therapies (19).

In this study, we investigated the features of TMB and explored the relationship of TMB with PTC recurrence. DEGs and immune cells were analyzed to explore the underlying mechanism of PTC recurrence.

\section{METHODS}

\section{Data Source}

The RNA-seq and single nucleotide polymorphism (SNP) data were all obtained from the TCGA pan-cancer cohort of the GDC data portal (HTTPS://portal.gdc.cancer.gov/). The RNA-seq comprised 512 tumor samples and 58 normal samples. Out of 512 tumor samples, 487 had complete SNP data, including simple nucleotide variation data (VarScan) and masked somatic mutation data. Basic clinical information included age, gender, AJCC-TNM stages, pathological subtype, survival outcomes, and follow-up time. The "maftools" software package, which could convert mutation data in Variant Call Format (VCF) format into visualization results (20), was used to describe the basic mutation characteristics of PTC samples. The mutation information of genes in each sample was displayed in the waterfall chart.

\section{TMB Calculation}

TMB generally refers to the number of somatic non-synonymous mutations per base pair $(\mathrm{Mb})$ in a specific gene group region based on whole-exome modeling calculations. We selected SNP data from genome sequencing to calculate TMB. In our study, the mutation frequency and the number of variants in each sample were calculated through the Perl script based on the JAVA8 platform, as described in the previous literature (21). Then the PTC patients were divided into two groups according to the cut-off point (the median of the TMB value).

\section{Estimation of TMB and Prognostic Analysis}

Then Kaplan-Meier analysis was utilized to compare the difference in RFS between the high and low TMB groups, evaluated by the log-rank test. Chi-square test was used to assess the differences of clinical characteristics between high and low TMB groups. Subsequently, the correlations of TMB levels with clinical characteristics were appraised. The Wilcoxon rank-sum test was used to compare the differences between the two groups of clinical variables. P-value $<0.05$ was considered statistically significant.

\section{DEGs and Functional Enrichment Analysis}

DEGs were selected based on the high and low TMB groups of PTC patients through the "limma" software package (22), with $\log 2 \mid$ fold change $\mid>1$ and False Discovery Rate $(F D R)<0.05$. The "heatmap" package was utilized to draw DEGs' heatmap plot, and the "vioplot" package drew the volcano plot. Then we employed the Gene Ontology (GO) analyses through the "clusterProfiler" package (23) to analyze the possible biological processes of the overlapping DEGs. We also applied gene set enrichment analysis (GSEA 4.0.1) to further explore the potential differences in PTC patients' biological functions.

\section{Estimation of Tumor-Infiltrating Immune Cells}

We employed the downloaded RNA-seq data through the deconvolution algorithm in the CIBERSORT website (http:// cibersort.stanford.edu/). CIBERSORT is a general calculation method that could combine support vector regression and prior knowledge of purified leukocyte subsets' composition profile to accurately estimate the composition of 22 immune cells in tumor tissues, and the method could replace flow cytometry or immunohistochemistry which is difficult to standardize (24-26). We subsequently identified the differences of 22 immune cells between the two groups of PTC patients. 
"Vioplot" package was employed to show the differential immune infiltrates between the two groups. The data analysis used the Wilcoxon rank-sum test. The threshold p-value $<0.05$ was the criterion for calculating the significance of samples.

\section{Survival Analysis}

Eighteen differential genes based on the two groups were identified. The RFS of these differential genes was analyzed according to the survival package of $\mathrm{R}$ software to find the more valuable prognostic biomarker. Receiver operating characteristic (ROC) and the area under the curve (AUC) were presented by utilizing the "survival ROC" package to confirm the prognostic value of clinical features. All data were tested by using an independent t-test, and the P-value was set to $<0.05$.

\section{RESULTS}

\section{Gene Mutation Profile of PTC}

The top 30 genes with a mutation frequency per sample were displayed, and different color annotations indicated the different types of mutations (Figure 1). The most common type of mutation in the summarized figure was a missense mutation (Figure 2A). SNPs occurred more often than insertions or deletions (Figure 2B). SNV was mainly based on $\mathrm{C}>\mathrm{T}$ base substitution in PTC (Figure 2C). Also, we calculated the median of PTC variants per sample (Figure 2D) as six and summarized the variant classifications based on the median and range (Figure 2E). The top 10 most common mutated genes of PTC were exhibited (Figure 2F), in which BRAF mutations were observed in $59 \%$ of cases, NRAS mutations in $8 \%$, HRAS mutations in $3 \%$, TG mutations in $3 \%$, and TTN mutations in $2 \%$. Finally, we showed the coexistence and exclusive correlation between mutant genes, where green represented coexistence and brown described a mutually exclusive relationship (Figure 2G). Moreover, we could see the BRAF mutation had the exclusive association with NRAS mutation or HRAS mutation.

\section{The Differences in Different Clinical Characteristics Between the High and Low TMB Groups}

We calculated the TMB of 487 PTC samples, which was the total number of mutations per million bases, ranging from 0.026 to 2.210 $\mathrm{Mut} / \mathrm{Mb}$, with a median of $0.211 \mathrm{Mut} / \mathrm{Mb}$. According to the median value of TMB, we divided the PTC patients into the high and low TMB groups. Then we compared the differences in different clinical characteristics between the high and low TMB groups, including age, gender, AJCC-TNM stages, pathological subtype, BRAF status, and survival outcomes (Table 1). Because some samples lack clinical data, the number of samples exhibited in Table 1 was not exactly complete. We could see that the high TMB group had a

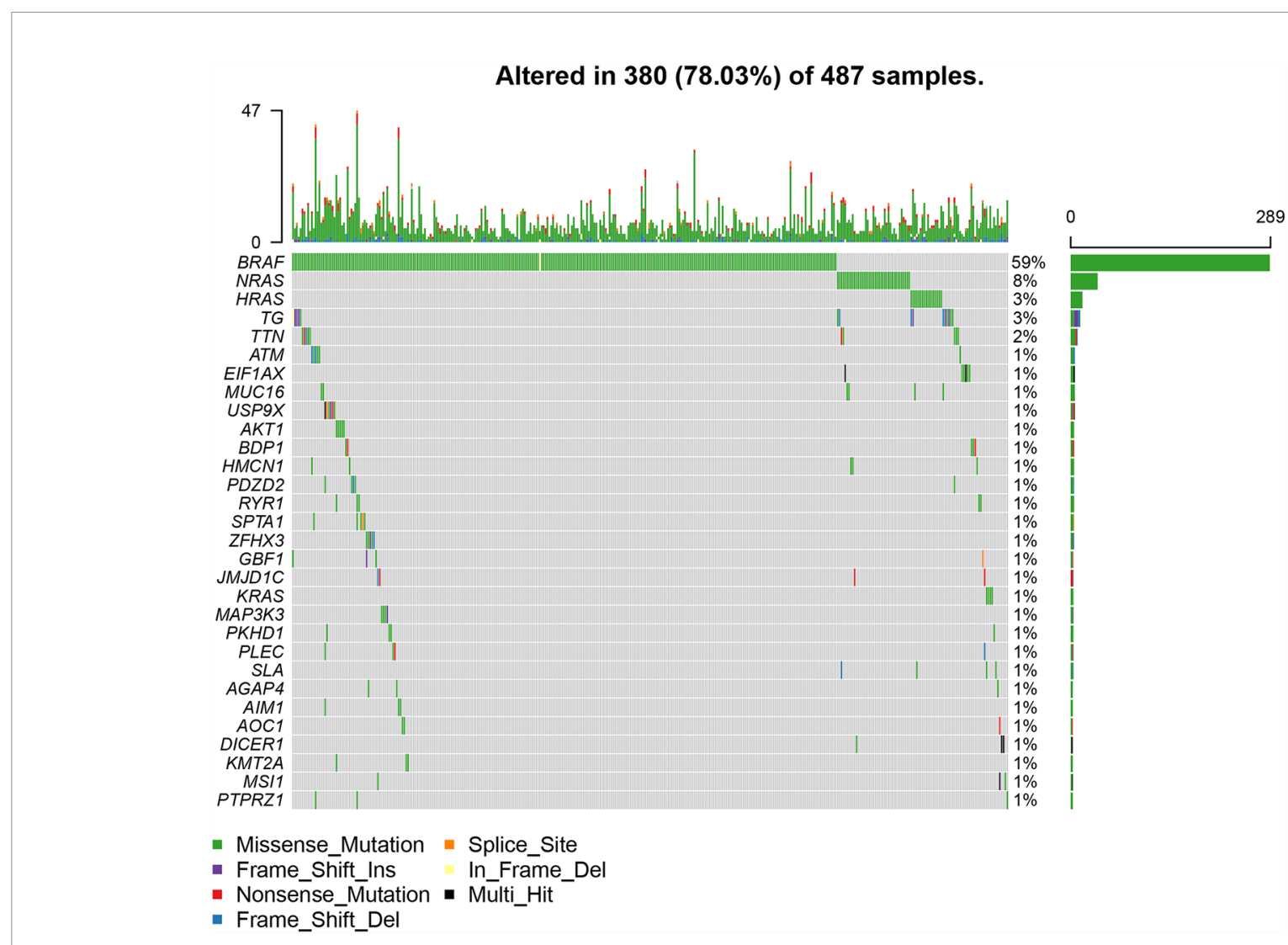

FIGURE 1 | The landscape of most common mutated genes in PTC. The waterfall chart showed the top 30 frequently mutated genes of PTC in the TCGA cohort. 

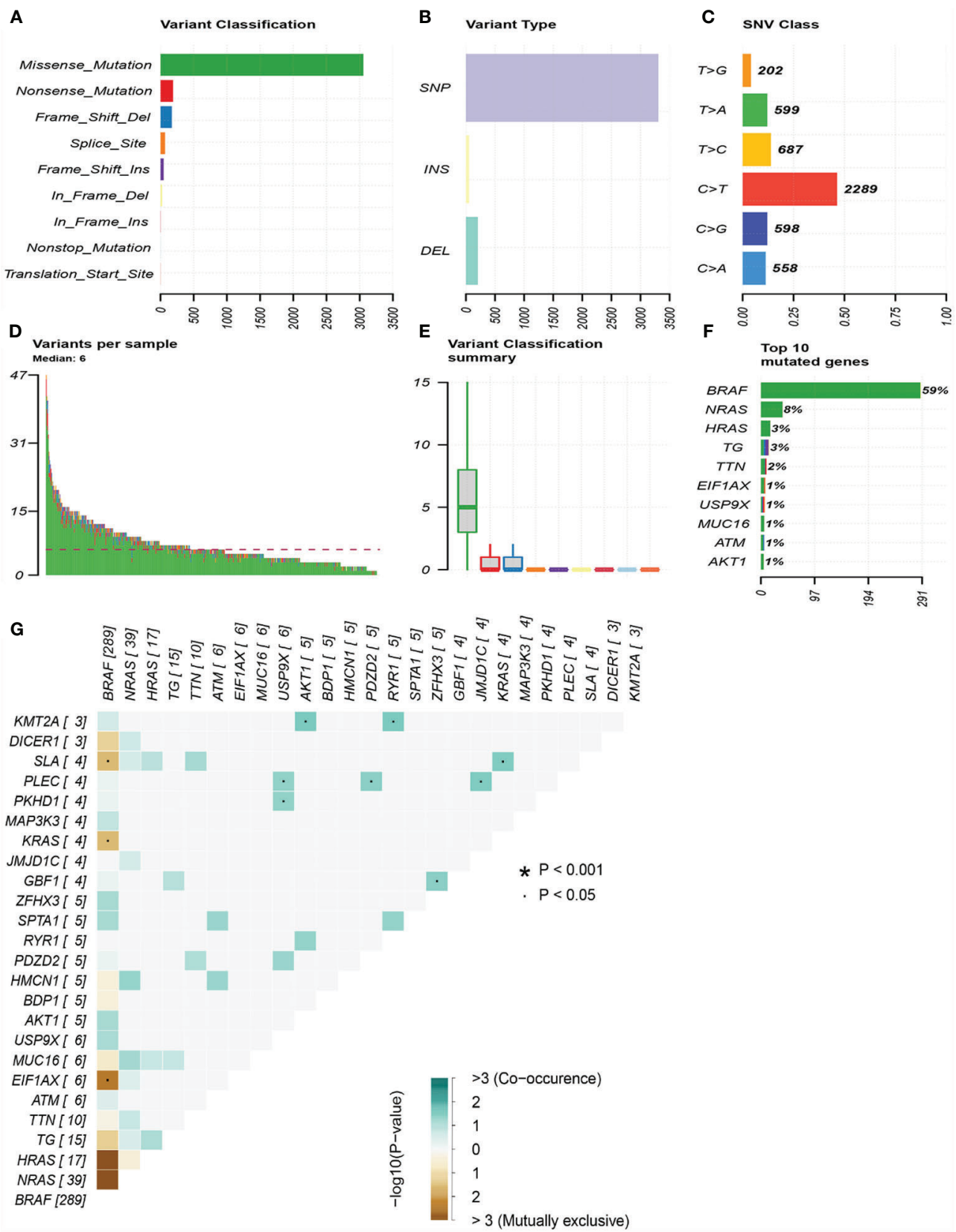

FIGURE 2 | Summary of mutation information in the TCGA thyroid cancer cohort. (A) Types of gene mutations. (B) Types of genome variation. (C) Types of single nucleotide changes. (D) The median number of mutations per sample. (E) Summary of gene mutation categories. (F) Top 10 common mutant genes. (G) Coexistence and exclusivity between mutant genes.

higher proportion of PTC patients with older than 55 years old, larger tumors, higher tumor stages, and higher recurrence rate.

\section{Lower TMB Predicted Better RFS Rate in PTC}

Patients in the high TMB group tended to have a lower recurrence-free survival rate than that in the low TMB group (Figure 3A). In addition, TMB levels were higher in patients older than 55 (Figure 3B) or male sex (Figure 3C). PTC in the T3-4 stages had higher TMB levels than that in the T1-2 stages (Figure 3D). And TMB levels in stages III-IV were increased significantly compared with that in stages I-II (Figure 3G). However, levels of TMB grouped by AJCC-N (Figure 3E), M stage (Figure 3F), histopathological type (Figure 3H), and BRAF status (Figure 3I) had no significant differences. Most importantly, the ROC AUC score of TMB was 0.780 , which was the most reliable indicator in the survival prediction of the model compared with age (AUC $=0.658)$, gender $(\mathrm{AUC}=0.421)$, AJCC- 
TABLE 1 | The differences of clinical characteristics between high and low TMB groups obtained from the TCGA cohort.

\begin{tabular}{|c|c|c|c|}
\hline Clinical characteristic & Low TMB (\%) & High TMB (\%) & $P$ value \\
\hline \multicolumn{4}{|l|}{ Age } \\
\hline$<55$ & $198(40.8)$ & 129 (26.6) & \\
\hline$>=55$ & $45(9.3)$ & $113(23.3)$ & $<0.001$ \\
\hline \multicolumn{4}{|l|}{ Gender } \\
\hline Female & $188(38.8)$ & $169(34.8)$ & \\
\hline Male & 55 (11.3) & $73(15.1)$ & 0.06 \\
\hline \multicolumn{4}{|l|}{ AJCC-T } \\
\hline $\mathrm{T} 1-2$ & 167 (34.4) & $130(26.8)$ & \\
\hline T3-4 & $76(15.7)$ & 111 (22.9) & 0.001 \\
\hline $\mathrm{TX}$ & $0(0)$ & $1(0.2)$ & \\
\hline \multicolumn{4}{|l|}{ AJCC-N } \\
\hline NO & 113 (23.3) & 106 (21.9) & \\
\hline N1 & 101 (20.9) & 116 (23.9) & 0.291 \\
\hline$N X$ & $29(6.0)$ & $20(4.1)$ & \\
\hline \multicolumn{4}{|l|}{ AJCC-M } \\
\hline $\mathrm{MO}$ & $138(28,5)$ & $135(27.8)$ & \\
\hline M1 & $3(0.6)$ & $6(1.2)$ & 0.498 \\
\hline$M X$ & $102(21.0)$ & 101 (20.8) & \\
\hline \multicolumn{4}{|l|}{ AJCC-Stage } \\
\hline Stages I-II & 242 (49.9) & 221 (45.6) & \\
\hline Stages II-IV & $1(0.2)$ & $21(4.3)$ & $<0.001$ \\
\hline \multicolumn{4}{|l|}{ Histopathology } \\
\hline Classical & $175(36.1)$ & 165 (34.0) & \\
\hline Follicular & $52(10.7)$ & $48(9.9)$ & \\
\hline Tall Cell & $13(2.7)$ & $23(4.7)$ & 0.202 \\
\hline Other & $2(0.4)$ & $7(1.4)$ & \\
\hline \multicolumn{4}{|l|}{ BRAF } \\
\hline Wild & $102(21.0)$ & $94(19.4)$ & \\
\hline Mutation & $140(28.9)$ & $149(30.7)$ & 0.437 \\
\hline \multicolumn{4}{|l|}{ Outcome } \\
\hline Free-recurrence & 221 (46.5) & $209(44)$ & \\
\hline Recurrence & $16(3.4)$ & $29(6.1)$ & 0.043 \\
\hline
\end{tabular}

Bold values refers to whether there is a difference in the number of people with different clinical characteristics between the high TMB group and the low TMB group through the chi-square test, and a $P$ value less than 0.05 has a statistical difference.

$\mathrm{T}(\mathrm{AUC}=0.668), \mathrm{N}(\mathrm{AUC}=0.566), \mathrm{M}(\mathrm{AUC}=0.611)$, tumor stage $(A U C=0.708)$, or BRAF status $(A U C=0.520)($ Figure $3 J)$.

\section{Identification of DEGs Based on High and Low TMB Groups}

Eighteen DEGs (Table 2) were identified from high and low TMB groups in the TCGA database, which were shown by heatmap (Figure 4A). Fourteen down-regulated and four upregulated genes in high TMB group were shown in the volcano plot (Figure 4B). Further, we explored the function of DEGs by GO analysis and found that DEG enrichment was mainly animal organ regeneration, extracellular matrix, rough endoplasmic reticulum, receptor antagonist activity, receptor inhibitor activity, and steroid hormone receptor activity (Figure 4C). The GSEA result was related to oxidative phosphorylation (OXPHOS), which demonstrated that the TMB in the high TMB group was positively correlated with OXPHOS level, while that in the low TMB group was the opposite (Figure 4D).

\section{IL37, SLC5A5, NR4A3, and ODF3L1 Associated With PTC Relapse}

We performed survival analysis on the 18 TMB related DEGs. Interleukin 37 (IL37), solute carrier family 5 member
(SLC5A5), nuclear receptor subfamily 4 group A member 3 (NR4A3) and outer dense fiber of sperm tails 3 like 1 (ODF3L1) had been determined to be correlated with RFS of PTC patients. The results showed that higher expression of IL37 in tumor tissues predicted worse prognosis outcomes (Figure 5A). Conversely, the other three genes with a higher level in tumor tissues predicted a better prognosis (Figures 5B-D). The relationship between the four DEGs and each clinicopathological factor per sample in the high and low TMB groups was shown by the heatmap (Figure 5E). We could observe that the high expression of IL37 was concentrated in clinical features with poor prognosis, while the SLC5A5, NR4A3, and ODF3L1 were concentrated in clinical features with good prognosis. We further confirmed the expression levels of the four differential expression genes according to the RNA-sequencing dataset from TCGA. The expression level of IL37 in the tumor was significantly higher than that in the normal tissues (Figure 6A), while SLC5A5, NR4A3, and ODF3L1 were highly expressed in normal tissues than those in tumors (Figures 6B-D).

\section{Differences in Immune Cell Infiltration Between High and Low TMB Groups}

Through the deconvolution algorithm on the CIBERSORT website, the relative scale of 22 immune cells was measured. PTC patients in the high TMB group had lower infiltration of $\mathrm{CD}^{+}{ }^{+} \mathrm{T}$ cells and $\mathrm{M} 1$ macrophages but higher gamma delta $\mathrm{T}$ cells than those in the low TMB group (Figure 7).

\section{DISCUSSION}

The previous study has shown that the Tumor Node Metastasis (TNM) staging guide cannot effectively predict the prognosis of patients with low-risk PTC during initial treatment (27). Herein, we defined the characteristics of TMB in PTC and compared $\mathrm{TMB}$ as the best recurrence predictor than other clinical features. Generally speaking, TMB is the number of somatic nonsynonymous mutations per base pair $(\mathrm{Mb})$ in a specific gene group region. Herein we selected SNP data which was similar to whole-exome sequencing to calculate TMB. However, the TMB values of patients with different cancer types are highly heterogeneous, and the high TMB thresholds screened out still show huge differences in different cancer types (28). Besides, since many of the original studies on TMB are based on wholeexome modeling calculations, it is usually visualized as the number of mutations in the coding region of the genome (exome) (29). In fact, in addition to point mutations that could produce neoantigens, frameshift mutations produced by insertions and deletions could also cause tumors to produce neoantigens, thereby changing the efficacy of immune checkpoint inhibitors (30). The TMB we calculated based on genome sequencing was caused by somatic non-synonymous mutations, and the changes in TMB which might be caused by other factors were not taken into account, such as insertions or 


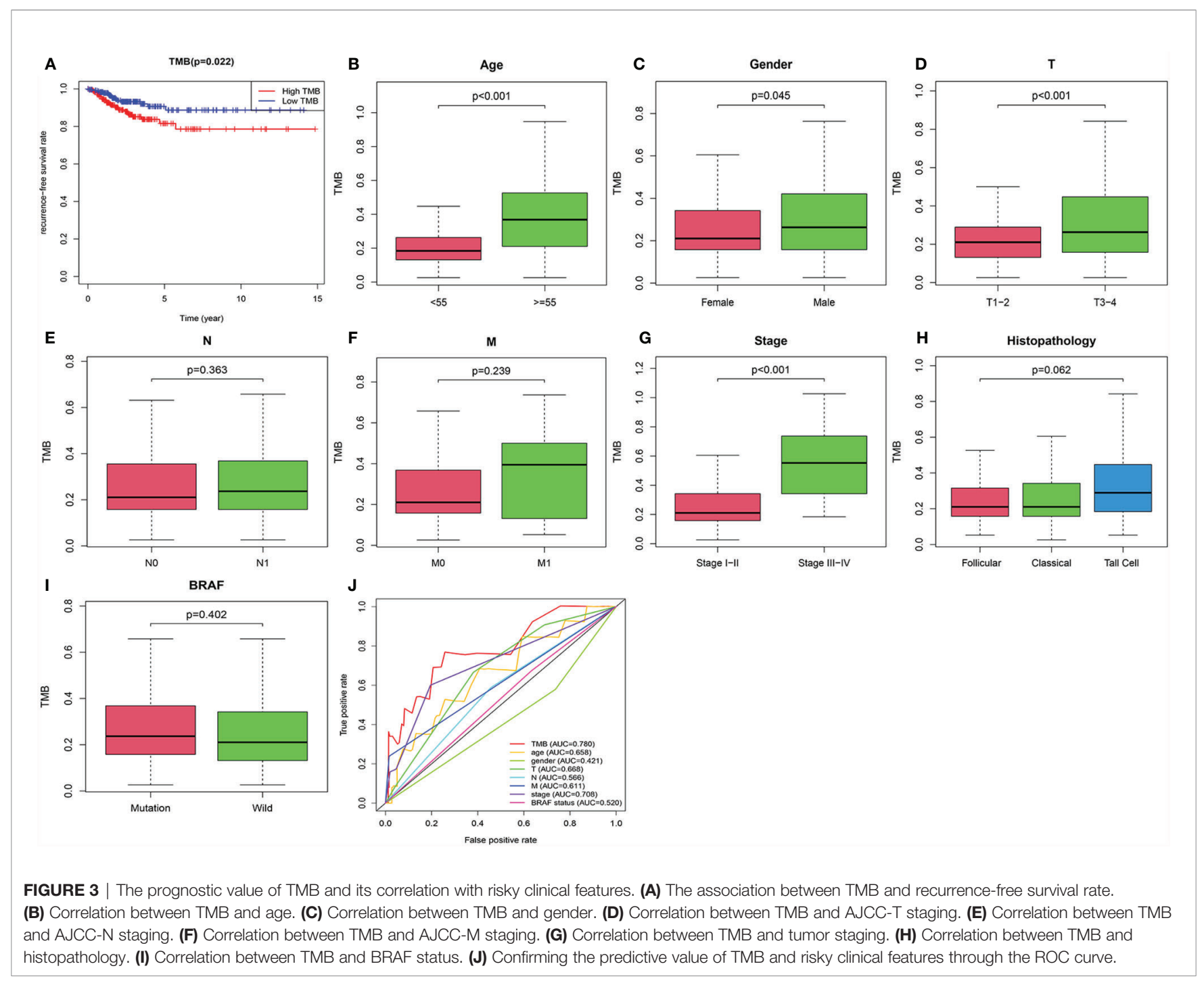

TABLE 2 | The DEGs between the high and low TMB groups.

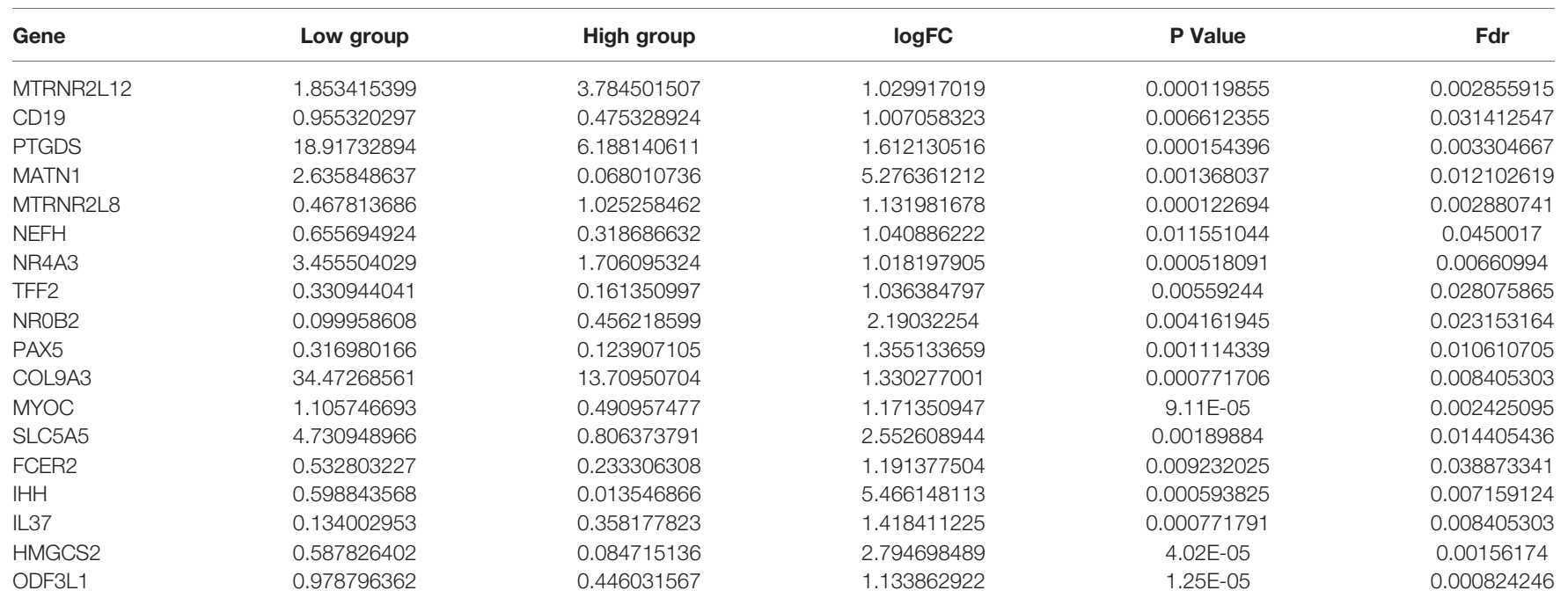


A

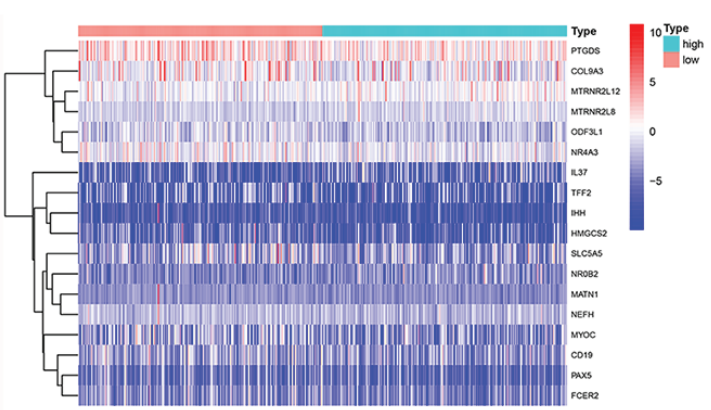

C

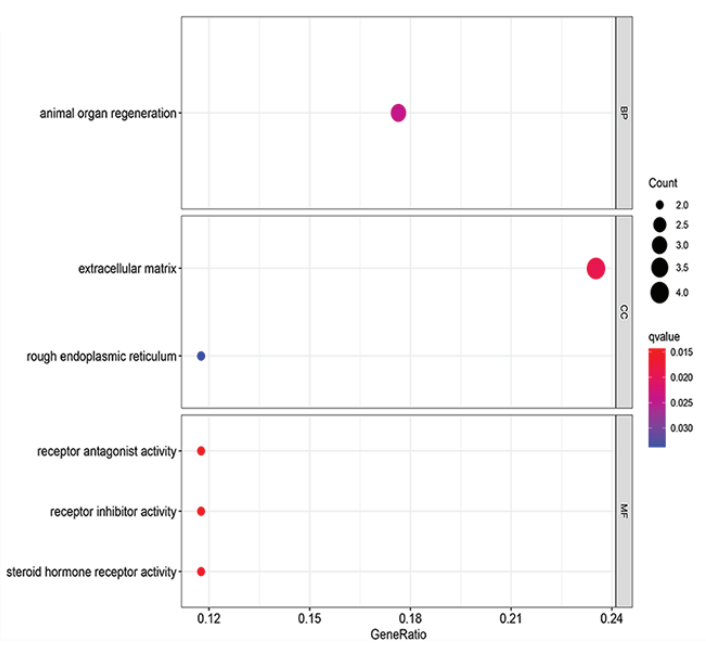

B

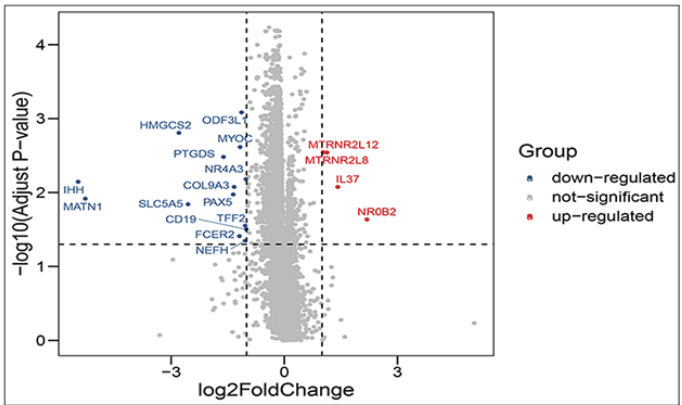

D

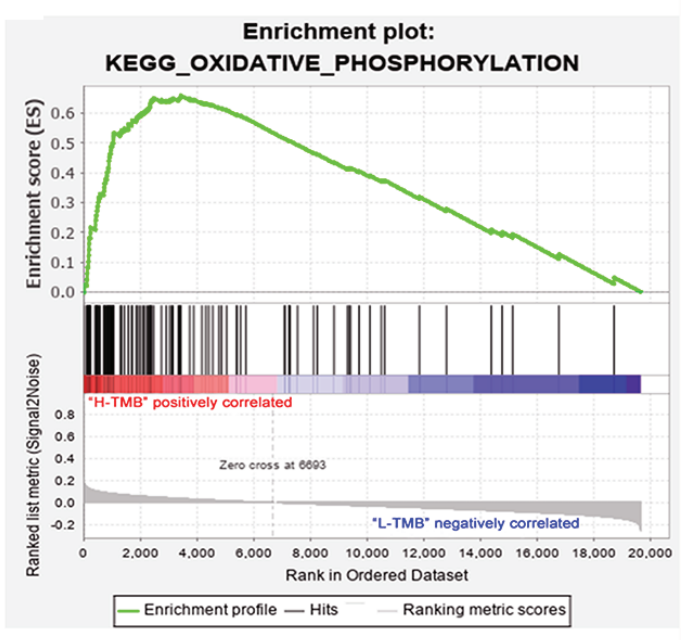

FIGURE 4 | Analysis of differentially expressed genes and functional pathways in the low and high TMB groups. (A) Eighteen DEGs were exhibited in the heatmap. (B) Fourteen down-regulated and four up-regulated genes of high TMB group shown in volcano plot. (C) GO analysis of TMB-related DEGs in PTC. (D) Gene set enrichment analysis (GSEA) between high and low TMB groups (the high TMB group was positively correlated with OXPHOS, while the low TMB group was negatively correlated with OXPHOS).

deletions. Therefore, in future, TMB detection still needs to consider many other interference factors.

GO analysis revealed that DEG enrichment was mainly involved in metabolic pathway. Also, patients in high TMB group showed poorer RFS for an increase in the level of OXPHOS. $\mathrm{CD}^{+} \mathrm{T}$ cells and M1 macrophages were decreased, which might be responsible for the recurrence of PTC with high TMB. Together these findings indicated that TMB in the tumor could help in effectively taking initial measures to monitor recurrence probability and avoid secondary surgery in the clinic. TMB has been reported to play important roles in many cancers (31). Higher TMB has more favorable RFS and is also a good predictor of response to ICPIs within a high microsatellite instability population in colorectal cancer $(32,33)$. Conversely, our study showed that PTC patients in the high TMB group had worse RFS (Figure 3A). Higher TMB was also associated with age older than 55, male, tumor size, and tumor staging (Figures 3B-D, G). The result was consistent with previous reports on clear cell renal cell carcinoma $(21,34)$ TMB had a rising trend in tall cell subtypes compared to classic and follicular types, but there might be no statistical difference due to insufficient sample size. BRAF ${ }^{\mathrm{V} 600 \mathrm{E}}$ mutation is significantly related to aggressive clinicopathological features and PTC relapse $(35,36)$. Furthermore, we also showed BRAF mutations as the most frequent and more prevalent in PTC than other mutations based on the TCGA data (Figure 1), which was consistent with previous reports (37). The interaction analysis of mutant genes showed that coexistence was more common than mutual exclusion (Figure 2G). However, compared with BRAF status only, TMB was a better predictor of relapse (Figure 3H). In general, ROC analysis showed the sensitivity and specificity of TMB were better than other clinical features. In our study, we found a significant risk factor for PTC recurrence and aggressiveness.

OXPHOS is up-regulated in many cancers, and its inhibitors with appropriate therapeutic index have new applications in 

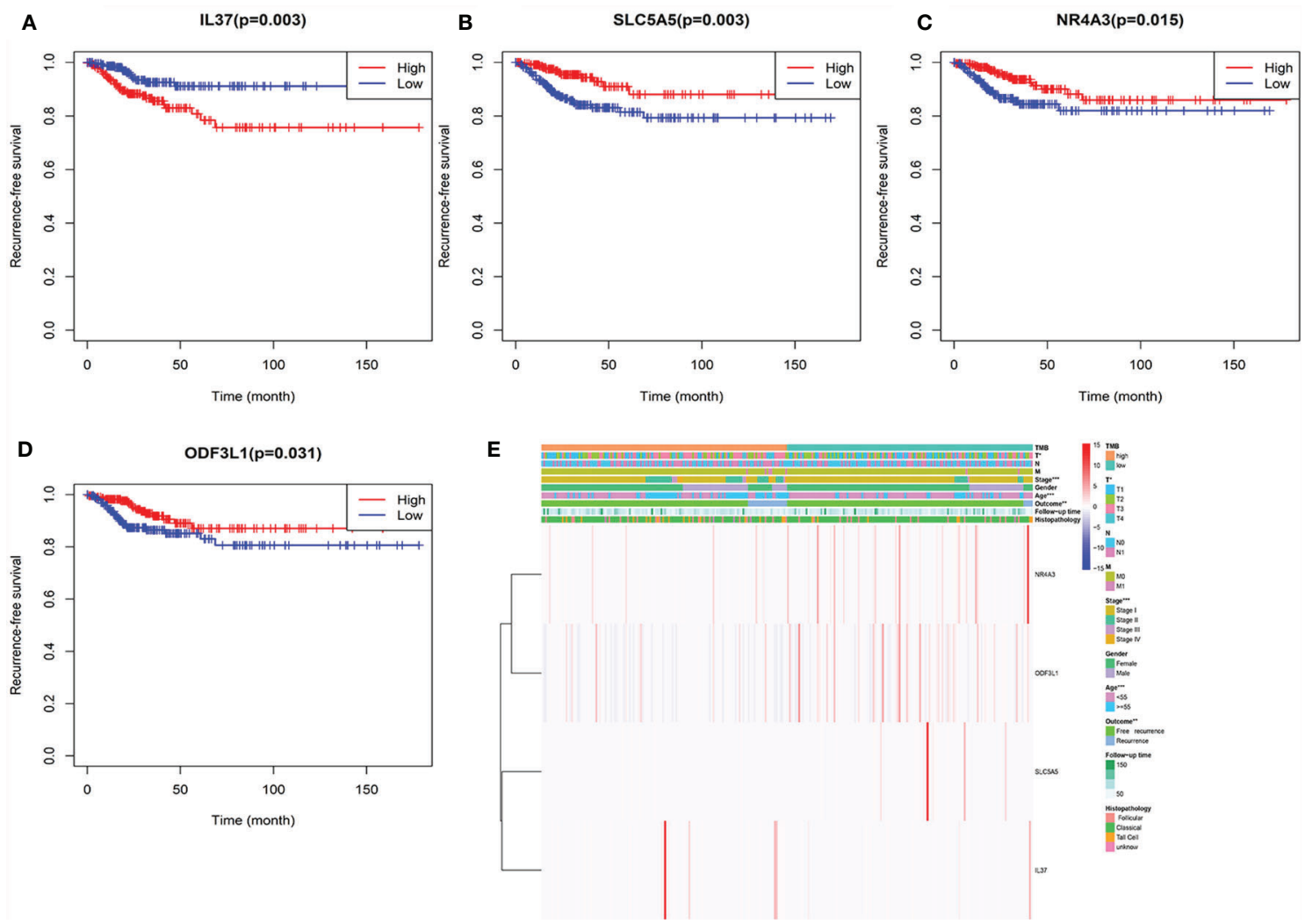

FIGURE 5 | Heatmap of the relationship between TMB related genes and clinical features. (A) Kaplan-Meier survival analysis of IL37. (B) Kaplan-Meier survival analysis of SLC5A5. (C) Kaplan-Meier survival analysis of NR4A3. (D) Kaplan-Meier survival analysis of ODF3L1. (E) The expression levels of the four genes and distribution of clinical features between the high and low TMB groups.

targeted cancer therapy, including thyroid cancer (38-40). GSEA results showed higher levels of OXPHOS in the tumors of patients in the high TMB group (Figure 4D), which might be a potential relapse mechanism in PTC with a higher mutation burden. In the immune microenvironment of the tumor, $\mathrm{CD} 8^{+} \mathrm{T}$ cells can recognize neoantigens on the surface of tumor to play an anti-tumor effect (41). Besides, high $\mathrm{CD}^{+} \mathrm{T}$ cell density was significantly associated with a good disease-free survival rate for PTC patients and with a reduced lymph node metastasis incidence (42), and M1 macrophages in the tumor microenvironment inhibit tumor growth (43-46). The low abundance of $\mathrm{CD}^{+} \mathrm{T}$ cells and M1 macrophages was also found in PTC with high TMB (Figure 7), which might also clarify the underlying reasons of high TMB causing relapse.

Furthermore, four TMB related differential genes were highly associated with prognosis (Figure 5). IL37 was a risky prognostic gene and might play a vital role in the development of PTC. The result was consistent with oral leukoplakia and oral squamous cell carcinoma (47). However, IL37 plays a protective role in most cancers, such as lung cancer (48) and colorectal cancer (49). At present, the function of IL37 in the carcinogenesis of PTC has not been explored. SLC5A5, which codifies sodium iodide symporter, plays an essential role in thyroid metabolism, mediating the active transport of iodine from the bloodstream into the follicular cells (50). Due to the decreased expression of SLC5A5, some patients with differentiated thyroid cancer were not sensitive to radioiodine therapy (51). NR4A3 is an orphan receptor and regulates the cellular function and inflammation reaction (52). Compared with thyroid follicular adenoma (FTA), NR4A3 was significantly down-regulated in follicular thyroid carcinoma (FTC), which led to reduced apoptosis factors (53). However, the function of NR4A3 in PTC is still unclear. As for ODF3L1, there is no research report yet; however, it is an excellent prognostic molecular marker in PTC. Though we detected four genes associated with PTC relapse, these genes' protein expression levels still need to be verified.

In conclusion, our results provided new information for the function of TMB in PTC. We showed that TMB was an independent prognostic factor to predict the recurrence of PTC and explained the possible mechanism. We also screened out genes related to relapse in PTC patients, which could become a new prognostic marker. At present, studies have shown that the 

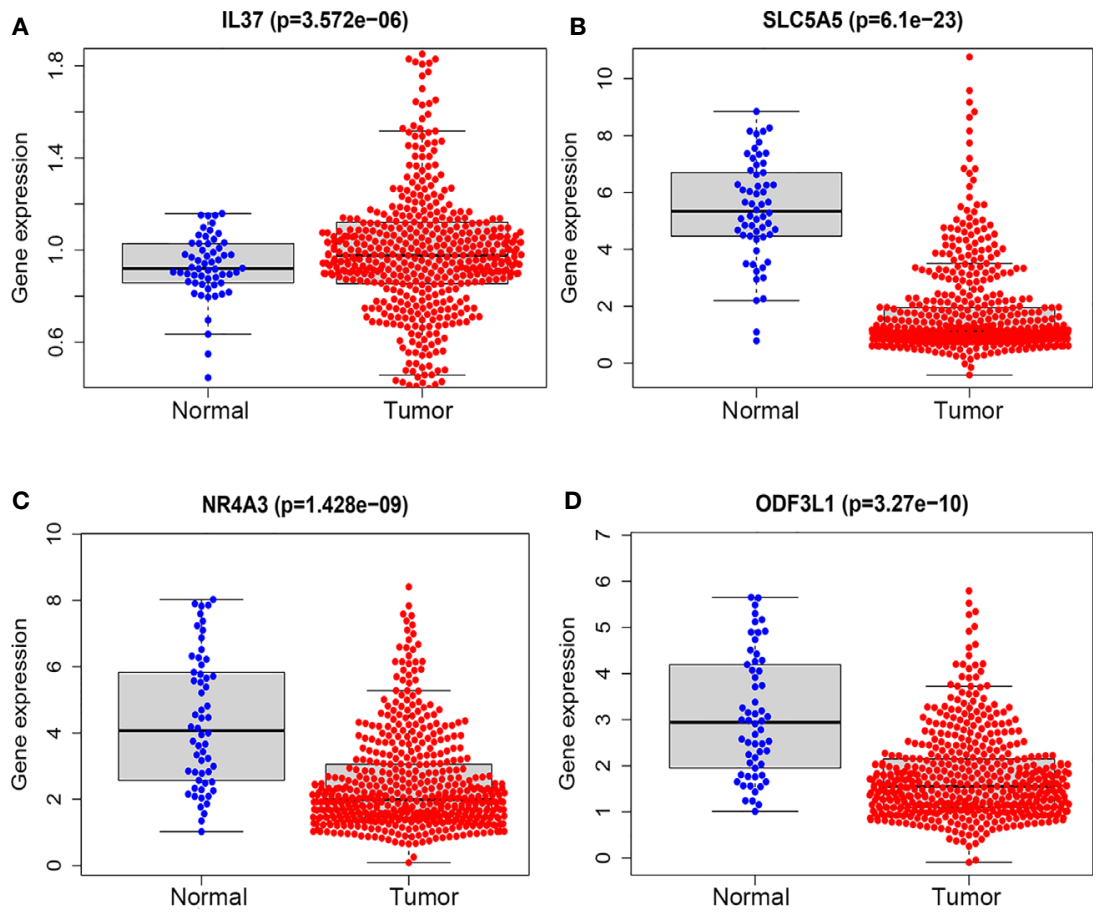

FIGURE 6 | TMB related prognostic genes in tumor and normal tissues of PTC based on TCGA database. (A) IL37. (B) SLC5A5. (C) NR4A3. (D) ODF3L1.

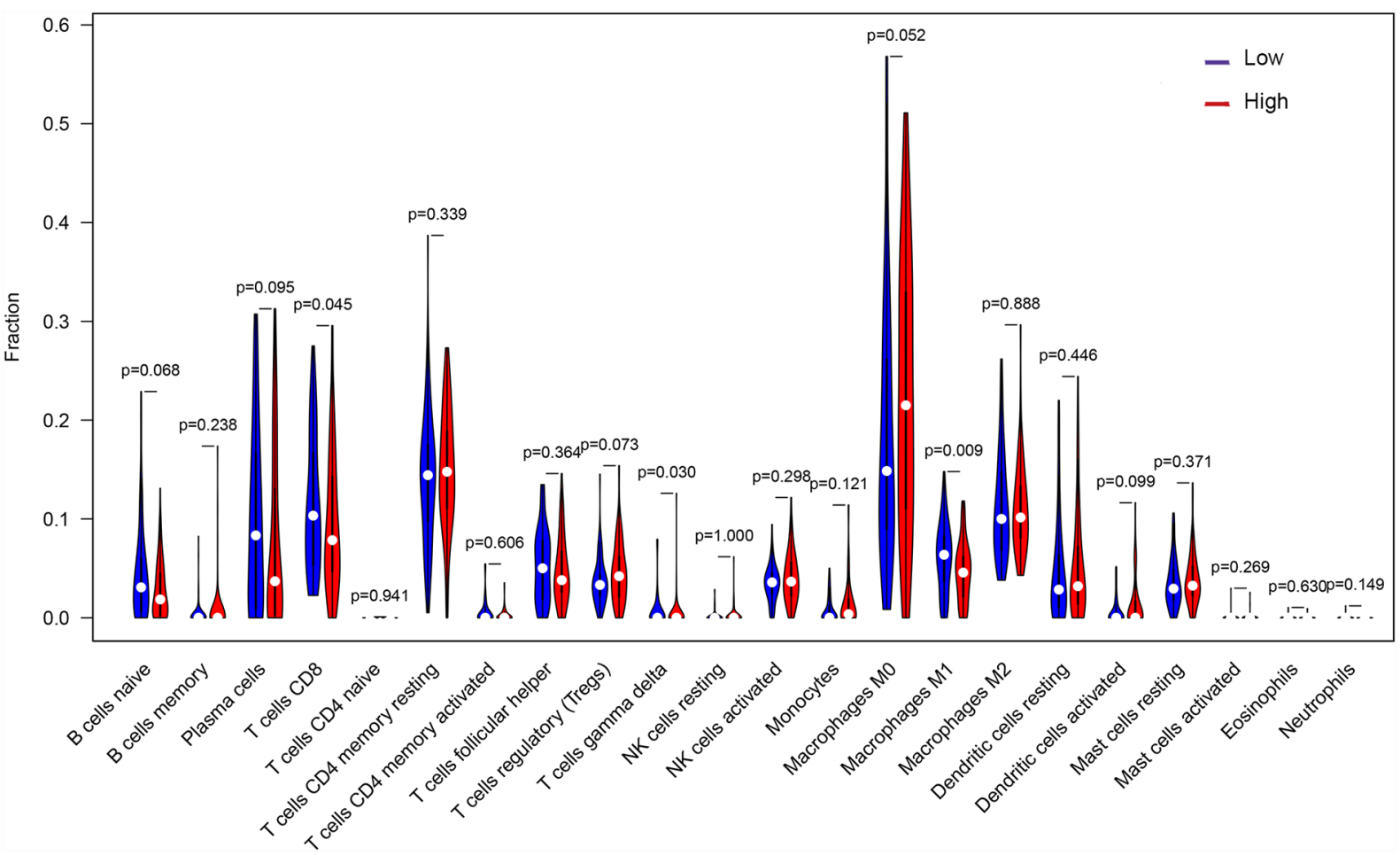

FIGURE 7 | Abundance of 22 immune cell infiltration between the high and low TMB groups. 
targeted sequencing panel combined with bioinformatics algorithm could effectively reduce the detection cost of TMB and sample size requirements (54). Nevertheless, our study was retrospective based on public databases, and the sample size was not large enough. The detailed mechanism needs to be further elucidated in future studies. In general, our findings strongly suggest that TMB is clinically meaningful for monitoring the recurrence to shorten the follow-up time of PTC.

\section{DATA AVAILABILITY STATEMENT}

The datasets presented in this study can be found in online repositories. The names of the repository/repositories and accession number(s) can be found below: https://portal.gdc. cancer.gov/exploration.

\section{ETHICS STATEMENT}

All data of the study were obtained from The Cancer Genome Atlas (TCGA) database and have obtained ethical approval.

\section{REFERENCES}

1. Cancer Genome Atlas Research, N. Integrated Genomic Characterization of Papillary Thyroid Carcinoma. Cell (2014) 159(3):676-90. doi: 10.1016/ j.cell.2014.09.050

2. Lim H, Devesa SS, Sosa JA, Check D, Kitahara CM. Trends in Thyroid Cancer Incidence and Mortality in the United States, 1974-2013. JAMA (2017) 317 (13):1338-48. doi: 10.1001/jama.2017.2719

3. Ahn D, Lee GJ, Sohn JH. Recurrence Following Hemithyroidectomy in Patients With Low- and Intermediate-Risk Papillary Thyroid Carcinoma. Br J Surg (2020) 107(6):687-94. doi: 10.1002/bjs.11430

4. Dong W, Horiuchi K, Tokumitsu H, Sakamoto A, Noguchi E, Ueda Y, et al. Time-Varying Pattern of Mortality and Recurrence From Papillary Thyroid Cancer: Lessons From a Long-Term Follow-Up. Thyroid (2019) 29(6):802-8. doi: $10.1089 /$ thy.2018.0128

5. Ding J, Wu W, Fang J, Zhao J, Jiang L. Male Sex is Associated With Aggressive Behaviour and Poor Prognosis in Chinese Papillary Thyroid Carcinoma. Sci Rep (2020) 10(1). doi: 10.1038/s41598-020-60199-9

6. Wang X, Cheng W, Liu C, Li J. Tall Cell Variant of Papillary Thyroid Carcinoma: Current Evidence on Clinicopathologic Features and Molecular Biology. Oncotarget (2016) 7(26):40792-9. doi: 10.18632/oncotarget.8215

7. Llamas-Olier AE, Cuéllar DI, Buitrago G. Intermediate-Risk Papillary Thyroid Cancer: Risk Factors for Early Recurrence in Patients With Excellent Response to Initial Therapy. Thyroid (2018) 28(10):1311-7. doi: 10.1089/thy.2017.0578

8. Kim HS, Choi JH, Lee JY, Kang J, Myung JK, Kim WH, et al. Downregulation of SMOC2 Expression in Papillary Thyroid Carcinoma and Its Prognostic Significance. Sci Rep (2020) 10(1):4853. doi: 10.1038/s41598-020-61828-Z

9. Sanjari M, Kordestani Z, Safavi M, Mashrouteh M, FekriSoofiAbadi M, Ghaseminejad A. Enhanced Expression of Cyclin D1 and C-myc, a Prognostic Factor and Possible Mechanism for Recurrence of Papillary Thyroid Carcinoma. Sci Rep (2020) 10(1):5100. doi: 10.1038/s41598-02061985-1

10. Jianyong L, Zhihui L, Rixiang G, Jingqiang Z. Using a Nomogram Based on Preoperative Serum Fibrinogen Levels to Predict Recurrence of Papillary Thyroid Carcinoma. BMC Cancer (2018) 18(1):390. doi: 10.1186/s12885-0184296-7

11. Enumah S, Fingeret A, Parangi S, Dias-Santagata D, Sadow PM, Lubitz CC, et al. Braf(V600e) Mutation is Associated With an Increased Risk of Papillary

\section{AUTHOR CONTRIBUTIONS}

MG, ZC, and BX conceived the study. QL, FS, and JF designed and revised the manuscript. MG, YL, SL, XG, FS, JF and WC analyzed the data. All authors contributed to the article and approved the submitted version.

\section{FUNDING}

This research was supported by Guangzhou medicine and healthcare technology projects (20211A011010) and Guangzhou Science and Technology Plan Project (202102080170).

\section{ACKNOWLEDGMENTS}

We would like to express our appreciation for the pre-dominant technical support of Chronic Disease Laboratory, Institutes for Life Sciences and School of Medicine, South China University of Technology. Also, we would like to thank The Cancer Genome Atlas (TCGA) database, which provided great help to our research.

Thyroid Cancer Recurrence. World J Surg (2020) 44(8):2685-91. doi: 10.1007/ s00268-020-05521-2

12. Kempf E, de Beyer JA, Cook J, Holmes J, Mohammed S, Nguyen TL, et al. Overinterpretation and Misreporting of Prognostic Factor Studies in Oncology: A Systematic Review. Br J Cancer (2018) 119(10):1288-96. doi: 10.1038/s41416-018-0305-5

13. Crow J, Samuel G, Godwin AK. Beyond Tumor Mutational Burden: Potential and Limitations in Using Exosomes to Predict Response to Immunotherapy. Expert Rev Mol Diagn (2019) 19(12):1079-88. doi: 10.1080/14737159.2020.1688144

14. Yarchoan M, Hopkins A, Jaffee EM. Tumor Mutational Burden and Response Rate to PD-1 Inhibition. N Engl J Med (2017) 377(25):2500-1. doi: 10.1056/ NEJMc1713444

15. Pleasance ED, Stephens PJ, O’Meara S, McBride DJ, Meynert D, Jones A, et al. A Small-Cell Lung Cancer Genome With Complex Signatures of Tobacco Exposure. Nature (2010) 463(7278):184-90. doi: 10.1038/nature08629

16. Zhang W, Edwards A, Flemington EK, Zhang K. Racial Disparities in Patient Survival and Tumor Mutation Burden, and the Association Between Tumor Mutation Burden and Cancer Incidence Rate. Sci Rep (2017) 7(1):13639. doi: 10.1038/s41598-017-13091-y

17. Hsu YC, Chang YH, Chang GC, Ho BC, Yuan SS, Li YC, et al. Tumor Mutation Burden and Recurrent Tumors in Hereditary Lung Cancer. Cancer Med (2019) 8(5):2179-87. doi: 10.1002/cam4.2120

18. Jaratlerdsiri W, Chan EKF, Gong T, Petersen DC, Kalsbeek AMF, Venter PA, et al. Whole-Genome Sequencing Reveals Elevated Tumor Mutational Burden and Initiating Driver Mutations in African Men With Treatment-Naive, High-Risk Prostate Cancer. Cancer Res (2018) 78(24):6736-46. doi: 10.1158/0008-5472.CAN-18-0254

19. Nandakumar V, Mills JR. The Now and Beyond of Tumor Mutational Burden as a Predictor of Response to Immune Checkpoint Inhibitors. Clin Chem (2019) 65(2):357. doi: 10.1373/clinchem.2018.295097

20. Mayakonda A, Lin DC, Assenov Y, Plass C, Koeffler HP. Maftools: Efficient and Comprehensive Analysis of Somatic Variants in Cancer. Genome Res (2018) 28(11):1747-56. doi: 10.1101/gr.239244.118

21. Zhang C, Li Z, Qi F, Hu X, Luo J. Exploration of the Relationships Between Tumor Mutation Burden With Immune Infiltrates in Clear Cell Renal Cell Carcinoma. Ann Transl Med (2019) 7(22):648. doi: 10.21037/atm.2019.10.84

22. Ritchie ME, Phipson B, Wu D, Hu Y, Law CW, Shi W, et al. Limma Powers Differential Expression Analyses for RNA-Sequencing and Microarray Studies. Nucleic Acids Res (2015) 43(7):e47. doi: 10.1093/nar/gkv007 
23. Yu G, Wang LG, Han Y, He QY, et al. clusterProfiler: An R Package for Comparing Biological Themes Among Gene Clusters. OMICS (2012) 16 (5):284-7. doi: 10.1089/omi.2011.0118

24. Chen B, Khodadoust MS, Liu CL, Khodadoust AM, Alizadeh AA, et al. Profiling Tumor Infiltrating Immune Cells With CIBERSORT. Methods Mol Biol (2018) 1711. doi: 10.1007/978-1-4939-7493-1_12

25. Desmedt C, Salgado R, Fornili M, Pruneri G, Van den Eynden G, Zoppoli G, et al. Immune Infiltration in Invasive Lobular Breast Cancer. J Natl Cancer Inst (2018) 110(7):768-76. doi: 10.1093/jnci/djx268

26. Gentles AJ, Newman AM, Liu CL, Bratman SV, Feng W, Kim D, et al. The Prognostic Landscape of Genes and Infiltrating Immune Cells Across Human Cancers. Nat Med (2015) 21(8):938-45. doi: 10.1038/nm.3909

27. Tuttle RM, Tala H, Shah J, Leboeuf R, Ghossein R, Gonen M, et al. Estimating Risk of Recurrence in Differentiated Thyroid Cancer After Total Thyroidectomy and Radioactive Iodine Remnant Ablation: Using Response to Therapy Variables to Modify the Initial Risk Estimates Predicted by the New American Thyroid Association Staging System. Thyroid (2010) 20 (12):1341-9. doi: 10.1089/thy.2010.0178

28. Samstein RM, Lee CH, Shoushtari AN, Hellmann MD, Shen R, Janjigian YY, et al. Tumor Mutational Load Predicts Survival After Immunotherapy Across Multiple Cancer Types. Nat Genet (2019) 51(2):202-6. doi: 10.1038/s41588018-0312-8

29. Chalmers ZR, Connelly CF, Fabrizio D, Gay L, Ali SM, Ennis R, et al. Analysis of 100,000 Human Cancer Genomes Reveals the Landscape of Tumor Mutational Burden. Genome Med (2017) 9(1):34. doi: 10.1186/s13073-017-0424-2

30. Turajlic S, Litchfield K, Xu H, Rosenthal R, McGranahan N, et al. Insertionand-Deletion-Derived Tumour-Specific Neoantigens and the Immunogenic Phenotype: A Pan-Cancer Analysis. Lancet Oncol (2017) 18(8):1009-21. doi: 10.1016/s1470-2045(17)30516-8

31. Romero D. TMB is Linked With Prognosis. Nat Rev Clin Oncol (2019) 16 (6):336. doi: 10.1038/s41571-019-0206-4

32. Lee DW, Han SW, Bae JM, Jang H, Han H, Kim H, et al. Tumor Mutation Burden and Prognosis in Patients With Colorectal Cancer Treated With Adjuvant Fluoropyrimidine and Oxaliplatin. Clin Cancer Res (2019) 25 (20):6141-7. doi: 10.1158/1078-0432.CCR-19-1105

33. Schrock AB, Ouyang C, Sandhu J, Sokol E, Jin D, Ross JS, et al. Tumor Mutational Burden is Predictive of Response to Immune Checkpoint Inhibitors in MSI-high Metastatic Colorectal Cancer. Ann Oncol (2019) 30 (7):1096-103. doi: 10.1093/annonc/mdz134

34. Zhang $\mathrm{C}, \mathrm{He} \mathrm{H}, \mathrm{Hu} \mathrm{X}$, Liu Ao, Huang Da, Xu Y, et al. Development and Validation of a Metastasis-Associated Prognostic Signature Based on SingleCell RNA-Seq in Clear Cell Renal Cell Carcinoma. Aging (2019) 11 (22):10183-202. doi: 10.18632/aging.102434

35. Geng J, Wang H, Liu Y, Tai J, Liu Y, Zhang J, et al. Correlation Between BRAF (V600E) Mutation and Clinicopathological Features in Pediatric Papillary Thyroid Carcinoma. Sci China Life Sci (2017) 60(7):729-38. doi: 10.1007/ s11427-017-9083-8

36. Xing M, Alzahrani AS, Carson KA, Shong YK, Kim TY, Viola D, et al. Association Between Braf V600E Mutation and Recurrence of Papillary Thyroid Cancer. J Clin Oncol (2015) 33(1):42-50. doi: 10.1200/jco.2014.56.8253

37. Landa I, Ibrahimpasic T, Boucai L, Sinha R, Knauf JA, Shah RH, et al. Genomic and Transcriptomic Hallmarks of Poorly Differentiated and Anaplastic Thyroid Cancers. J Clin Invest (2016) 126(3):1052-66. doi: 10.1172/JCI85271

38. Ashton TM, McKenna WG, Kunz-Schughart LA, Higgins GS, et al. Oxidative Phosphorylation as An Emerging Target in Cancer Therapy. Clin Cancer Res (2018) 24(11):2482-90. doi: 10.1158/1078-0432.CCR-17-3070

39. Sica V, Bravo-San Pedro JM, Stoll G, Kroemer G, et al. Oxidative Phosphorylation as a Potential Therapeutic Target for Cancer Therapy. Int J Cancer (2019) 146(1):10-7. doi: 10.1002/ijc.32616

40. Thakur S, Daley B, Gaskins K, Vasko VV, Boufraqech M, Patel D, et al. Metformin Targets Mitochondrial Glycerophosphate Dehydrogenase to Control Rate of Oxidative Phosphorylation and Growth of Thyroid Cancer
In Vitro and In Vivo. Clin Cancer Res (2018) 24(16):4030-43. doi: 10.1158/ 1078-0432.ccr-17-3167

41. Reading JL, Galvez-Cancino F, Swanton C, Lladser A, Peggs KS, Quezada SA, et al. The Function and Dysfunction of Memory CD8(+) T Cells in Tumor Immunity. Immunol Rev (2018) 283(1):194-212. doi: 10.1111/imr.12657

42. Aghajani MJ, Yang T, McCafferty CE, Graham S, Wu X, Niles N. Predictive Relevance of Programmed Cell Death Protein 1 and Tumor-Infiltrating Lymphocyte Expression in Papillary Thyroid Cancer. Surgery (2018) 163 (1):130-6. doi: 10.1016/j.surg.2017.04.033

43. Paul S, Chhatar S, Mishra A, Lal G. Natural Killer T Cell Activation Increases Inos(+)CD206(-) M1 Macrophage and Controls the Growth of Solid Tumor. J Immunother Cancer (2019) 7(1):208. doi: 10.1186/s40425019-0697-7

44. Travers M, Brown SM, Dunworth M, Holbert CE, Wiehagen KR, Bachman KE. DFMO and 5-Azacytidine Increase M1 Macrophages in the Tumor Microenvironment of Murine Ovarian Cancer. Cancer Res (2019) 79 (13):3445-54. doi: 10.1158/0008-5472.CAN-18-4018

45. Cao M, Yan H, Han X, Weng L, Wei Q, Sun X, et al. Ginseng-Derived Nanoparticles Alter Macrophage Polarization to Inhibit Melanoma Growth. J Immunother Cancer (2019) 7(1):326. doi: 10.1186/s40425-019-0817-4

46. Ren F, Fan M, Mei J, Wu Y, Liu C, Pu Q, et al. Interferon-Gamma and Celecoxib Inhibit Lung-Tumor Growth Through Modulating M2/M1 Macrophage Ratio in the Tumor Microenvironment. Drug Des Devel Ther (2014) 8. doi: 10.2147/DDDT.S66302

47. Lin L, Wang J, Liu D, Liu S, Xu H, Ji N, et al. Interleukin-37 Expression and its Potential Role in Oral Leukoplakia and Oral Squamous Cell Carcinoma. Sci Rep (2016) 6. doi: 10.1038/srep26757

48. Ge G, Wang A, Yang J, Chen Y, Yang J, Li Y, et al. Interleukin-37 Suppresses Tumor Growth Through Inhibition of Angiogenesis in Non-Small Cell Lung Cancer. J Exp Clin Cancer Res (2016) 35(1). doi: 10.1186/s13046-016-0293-3

49. Zhu B, Luo J, Jiang Y, Yu L, Liu M, Fu J. Prognostic Significance of Nomograms Integrating IL-37 Expression, Neutrophil Level, and MMR Status in Patients With Colorectal Cancer. Cancer Med (2018) 7(8):368294. doi: $10.1002 / \mathrm{cam} 4.1663$

50. Spitzweg C, Morris JC. Genetics and Phenomics of Hypothyroidism and Goiter Due to NIS Mutations. Mol Cell Endocrinol (2010) 322(1-2):56-63. doi: 10.1016/j.mce.2010.02.007

51. Dohán O, Baloch Z, Bánrévi Z, Livolsi V, Carrasco N. Rapid Communication: Predominant Intracellular Overexpression of the $\mathrm{Na}(+) / \mathrm{I}(-)$ Symporter (NIS) in a Large Sampling of Thyroid Cancer Cases. J Clin Endocrinol Metab (2001) 86(6):2697-700. doi: 10.1210/jcem.86.6.7746

52. Prince LR, Prosseda SD, Higgins K, et al. NR4A Orphan Nuclear Receptor Family Members, NR4A2 and NR4A3, Regulate Neutrophil Number and Survival. Blood (2017) 130(8):1014-25. doi: 10.1182/blood-2017-03-770164

53. Borup R, Rossing M, Henao R, Yamamoto Y, Krogdahl A, Godballe C, et al. Molecular Signatures of Thyroid Follicular Neoplasia. Endocr Relat Cancer (2010) 17(3):691-708. doi: 10.1677/ERC-09-0288

54. Zehir A, Benayed R, Shah RH, Syed A, Middha S, Kim HR, et al. Mutational Landscape of Metastatic Cancer Revealed From Prospective Clinical Sequencing of 10,000 Patients. Nat Med (2017) 23(6):703-13. doi: 10.1038/ nm.4333

Conflict of Interest: The authors declare that the research was conducted in the absence of any commercial or financial relationships that could be construed as a potential conflict of interest.

Copyright (c) 2021 Guo, Chen, Li, Li, Shen, Gan, Feng, Cai, Liu and Xu. This is an open-access article distributed under the terms of the Creative Commons Attribution License (CC BY). The use, distribution or reproduction in other forums is permitted, provided the original author(s) and the copyright owner(s) are credited and that the original publication in this journal is cited, in accordance with accepted academic practice. No use, distribution or reproduction is permitted which does not comply with these terms. 